Tome 42, no 12. - Juin 1935.

Mitgeteilt an Der Generalversammlung Der Schweizerischen zoologischen Gesellschaft, in Freiburg, den 16. und 17. März 1935.

\title{
Zirkus-Dressuren und Tierpsychologie ${ }^{1}$
} von

\section{H. HEDIGER}

(Zoologische Anstalt der Universität Basel).

Wer von der Zoologie her an die Tierpsychologie herantritt, der sieht deren erste Aufgabe darin, die psychischen Aeusserungen der Tiere menschlichem Verständnis zugänglich zu machen und die psychischen Fähigkeiten und Eigenarten der einzelnen Formen zu beschreiben und zu analysieren. Die auch nur einigermassen befriedigende Bewältigung dieser Aufgabe bedarf naturgemäss eines sehr grossen Zeitraumes; gegenwärtig befinden wir uns in den ersten Anfängen dazu. Dabei fällt aber jetzt schon auf, dass hier ein gewisses Missverhältnis besteht, ein Missverhältnis in der Bearbeitung naheliegender, handgreiflicher Fragen einerseits und entfernter, etwas gesuchter Probleme andererseits. Diese Zustände erinnern ein wenig an eine zoologische Systematik, die mit der Beschreibung der Protozoen und niedersten Wirbellosen beginnen würde und die auffälligsten Grossformen unserer nächsten Umgebung zunächst unbeachtet liesse.

Dieser Vergleich ist nicht sehr übertrieben. Wir besitzen - an sich zweifellos sehr begrüssenswerte und wertvolle - Arbeiten ernsthafter Forscher z. B. über die Intelligenz des Regenwurmes, über das Gedächtnis der Schnecke, über die Aufmerksamkeit bei der Mücke, usw. Aber wir finden nirgends eine psychologische Charakterisierung auch nur der allerauffälligsten unserer Zoo- und Zirkus-Tiere, wie z. B. von Löwe, Bär, Wolf, Giraffe, Seelöwe, Tiger, Hyäne, etc. Ja sogar über ein in theoretischer wie in praktischer Hinsicht so ausserordentlich wichtiges Geschöpf, wie der

1 Es handelt sich bei dem vorliegenden Aufsatz lediglich um eine vorläufige Mitteilung, die zunächst nur einige Punkte vom Grundsätzlichen zu diesem Thema aufzeigen möchte. Eine ausführlichere Darstellung des Gegenstandes wird später erscheinen.

Rev. Suisse de Zool., T. 42, 1935. 
Elefant eines ist, vermag uns die wissenschaftliche Tierpsychologie so gut wie nichts zu sagen.

Damit diese merkwürdigen Verhältnisse überhaupt verstanden werden können, muss auf zwei Tatsachen hingewiesen werden: Das Beobachten und Experimentieren mit solchen Tieren, die sich in jedem Laboratorium auf beschränktem Raum mit wenig Aufwand halten lassen, hat begreiflicherweise den Vorzug gegenüber der schwierigen und kostspieligen Haltung von Grosstieren. Die starke Bevorzugung kleiner und einfacher Formen durch die Tierpsychologie ist also z. T. durch diese Aeusserlichkeit begründet. Als vielleicht ebenso wichtiges Moment mag dabei aber auch die Ueberlegung mitgespielt haben, dass der Inangriffnahme der höchststehenden Tiere, deren psychische Organisation den höchsten Grad der Komplikation aufweist, die Untersuchung der einfacher organisierten Geschöpfe vorauszugehen hat, um auf der sicheren, am Einfachen gewonnenen Basis allmählich zum Komplexen überzugehen.

Ausser diesem rein äusserlichen und dem eben genannten logischen Grund muss aber wohl noch ein anderer, nämlich ein mehr subjektiver, dafür verantwortlich gemacht werden, dass die Dressur-Phänomene der Zirkustiere von der Tierpsychologie bis dahin vollständig vernachlässigt wurden: es scheint beinahe, als ob es die wissenschaftliche Tierpsychologie für sozusagen unter ihrer Würde gehalten hätte, sich mit Zirkus-Dingen zu beschäftigen. So übersah sie offenbar die zahlreichen Probleme, die in Wirklichkeit hier stecken. Wenn es aber eine Tierpsychologie überhaupt gibt, dann hat sie auch die Aufgabe, sich der einschlägigen Phänomene im Zirkus anzunehmen - ganz abgesehen davon, dass deren nähere Erforschung auch aus praktischen Gründen in verschiedenster Hinsicht von erheblichem Interesse ist.

In Wirklichkeit vermag der Zirkus der Tierpsychologie eine reiche Fülle interessanten Materials zu bieten. Die ganze, von der Tierpsychologie bis dahin geleistete Arbeit baut sich auf eine verschwindend kleine Anzahl von Formen auf (unter denen - wie wir gesehen haben - viele der auffälligsten Grosstiere fehlen) und zu einem beträchtlichen Teil noch auf Haustiere (weisse Maus, Ratte, Meerschweinchen, Huhn, Pferd, Hund usw.). Nun sind aber die Schlüsse, die wir aus dem Verhalten von Haustieren ziehen, deswegen mit ganz besonderer Vorsicht aufzunehmen, weil 
wir nichts wissen von den sicher sehr starken psychischen Einwirkungen des Domestikationsprozesses auf die ursprüngliche Wildform. Wir besitzen heute noch keine Psychologie der Domestikation - und diese wäre doch die allererste Voraussetzung für jedes tierpsychologische Arbeiten mit Haustieren! Wir sind daher z. T. auf die Untersuchung des Verhaltens von Wildtieren, wie wir sie eben im Zirkus finden, geradezu angewiesen; ihre durch das Gefangenleben bedingte Abweichung vom ursprünglichen Zustand kann zum mindesten noch weit besser übersehen werden (vgl. meine Arbeit über Zahmheit) als die viel grössere Abweichung der Haustiere von ihren Stammformen in psychischer Hinsicht. Anderseits bietet vielleicht gerade die Untersuchung der Gefangenschaftsbedingungen, in denen die Wildtiere im Zirkus gehalten werden, Einblicke in erste Anfänge von Domestikationserscheinungen.

Ein hübsches Beispiel dafür, dass die Untersuchung des Verhaltens von Wildtieren dem der Haustiere logischerweise vorauszugehen hat, zeigen uns die interessanten Forschungen der Drs. Menzel und D. Müllers über die sogen. Wesensgrundlagen beim Haushund. Es handelt sich dabei z. B. darum, ob es beim Hund einen Schutztrieb gibt, im Sinne einer ererbten Bereitschaft, dem Herdengenossen beizustehen. «Zur Beantwortung dieser Frage müssen wir ... wiederum uns der wilden Stammform fast aller Hunderassen, dem Wolfe, zuwenden ", sagt Müller. Sicher wäre hier neben den sehr schwierigen Beobachtungen aus freier Wildbahn auch das Verhalten dressierter Zirkuswölfe sehr aufschlussreich gewesen, wenn sich jemand die Mühe genommen hätte, über diese selten gezeigten Tiere im Zirkus einiges festzuhalten.

Die psychologische Charakterisierung einer Tierart lediglich auf Grund von Beobachtungen im Freileben - die wohl am schwierigsten von allen anzustellen sind - würde natürlich nur ein unvollständiges Bild geben. Es müssen notwendigerweise auch die verschiedenen Zustände des Gefangenlebens, der Wildheit, Eingewöhntheit, Zahmheit, Dressiertheit mitberücksichtigt werden (vgl. die Arbeit über Zahmheit). Was nun diesen letzten Punkt, die Dressiertheit, anbetrifft, der aus bestimmten Gründen ein ganz besonderes Interesse zukommt, so steht ihr die Tierpsychologie einstweilen wirklich ziemlich hilflos gegenüber.

Hier soll zunächst nur ganz allgemein auf das hingewiesen werden, 
was die Tierpsychologie grundsätzlich im Zirkus zu untersuchen hat. Es handelt sich dabei in der Hauptsache um folgende vier Punkte:

1. $\mathrm{D}$ a s $\mathrm{T}$ i e $\mathrm{r}$ a $\mathrm{n} \mathrm{si} \mathrm{ch}$, in seinen individuellen und artlichen Eigenarten. Hier liegt das Arbeitsgebiet der Speziellen Tierpsychologie (welche die Besonderheiten der einzelnen Formen untersucht) und der tierischen Individualpsychologie.

2. D a s Verhaiten des Mensehen gegenüber $\mathrm{d}$ e $\mathrm{m}$ T i e r. Hierhin gehört die Untersuchung der verschiedenen Dressurmethoden und ihrer Wirkung auf das einzelne Individuum, wie auch auf die einzelne Rasse, Art und höhere Einheit. Ferner wäre da zu prüfen das Problem der Affekt-Uebertragung, die Frage nach suggestiven Beeinflussungsmöglichkeiten, nach der Bedeutung des menschlichen Blickes, usw.

3. D a s Verhalten der Tiere unter sich, und zwar sowohl artgleicher Individuen als auch sogen. gemischter Gruppen. Hier ist das Arbeitsfeld der tierischen Charakterologie und Sozialpsychologie.

4. D a s Verhalten des Tieres gegen über dem $\mathrm{M}$ e n s c hen. Hier müsste $u$. a. die zuweilen sehr starke und sehr dauerhafte Bindung des Tieres an den Menschen (Dompteur) untersucht werden, das sogen. Kumpanen-Verhältnis UEхк ÜLLS bezw. das Meuten-Verhältnis der Drs. Menzel. Der Dompteur, wird ja nicht selten auch einbezogen in die soziale Struktur, in die Rangordnung seiner Tiergruppe.

Was nun die Erforschung der hier in erster Linie interessierenden Dressur-Phänomene ${ }^{1}$ anbetrifft, so scheint es auf den ersten Blick ein sehr einfaches Mittel zu geben zur Ueberbrückung der erheblichen Diskrepanz, welche besteht einerseits zwischen der Aufgabe des Untersuchers, die Dressurphänomene wissenschaftlich zu analysieren und einzuordnen und andererseits seiner persönlichen, praktischen Erfahrung im direkten Umgang mit Zirkustieren. Dieses einfache Mittel scheint bei oberflächlicher Betrachtung darin zu bestehen, die Dompteure auszufragen, denn diese beweisen ja durch ihre blosse Existenz, dass sie allerhand von den zu unter-

1 Inbezug auf den Ausdruck Dressur herrscht in der Literatur eine grosse Begriffsverwirrung, auf die erst in der angekündeten Arbeit eingegangen werden kann. 
suchenden Phänomenen verstehen. Diese Methode ist indessen völlig unzulässig, sofern sie ausschliesslich Verwendung findet; sie wäre ebenso verfehlt, wie die, z. B. einen Wasserschmecker auszufragen, um sich von ihm über die physikalischen und physiologischen Grundlagen des Wünschelrutenproblems unterrichten zu lassen. Die Methode der Befragung darf nur in zweiter Linie und immer nur mit grösster Vorsicht angewendet werden. Wie der Wasserschmecker, so arbeitet auch der Dompteur hauptsächlich gefühlsmässig, ohne sich im allgemeinen über seine Handlungen lange Rechenschaft zu geben. Dem Dompteur fehlt naturgemäss die objektive und kritische Einstellung - dem wissenschaftlichen Untersucher dagegen die persönliche Erfahrung, die hier ebenso wichtig ist. Das eine scheint das andere auszuschliessen.

Bei genauerem Hinsehen zeigt es sich aber doch, dass uns für die wissenschaftliche Untersuchung der Dressur im Zirkus folgendes Material zur direkten Beobachtung zur Verfügung steht:

a). Di e regulären Vorführungen. Diese zeigen uns die Tiere im Zustande der Dressiertheit, also wenn ihnen die erlernten Dressur-Leistungen bereits geläufig sind. Wir haben hier durch fortgesetztes Beobachten und Protokollieren einer Reihe aufeinanderfolgender Vorführungen die Möglichkeit, einmal die ganze Variationsbreite des Verhaltens der Tiere bei ihrer "Arbeit " zu erfassen, was u. a. zur Beurteilung der Frage nach der Plastizität der Aufgabenerfassung sehr aufschlussreich ist. Ausserdem können wir hier die Wirkung verschiedener äusserer Einflüsse wie z. B. Witterung, Temperatur (die eine sehr bedeutende Rolle spielt), Tageszeit, Publikumsverhalten, usw. statisch feststellen. Auch einen Einblick in die Wirkung besonderer Stimmungen auf Seiten der Tiere sowohl als auch des Dompteurs auf die Dressurleistungen können wir hier gewinnen. Dagegen sagen uns die in fertig dressiertem Zustand vorgeführten Tiere nichts aus über die Dressurmethoden, die bei ihnen angewendet wurden, über die Genese der Dressurleistungen, über die Dressur-Dauer, usw. Um darüber Beobachtungen anzustellen, steht uns im Zirkus eine zweite Gruppe von Erscheinungen zur Verfügung:

b) Di e Proben. Diese zeigen uns die Tiere in der Regel im Zustande der Dressur, die ja einen noch nicht abgeschlossenen, laufenden Prozess darstellt. Hier haben wir nun die Möglichkeit, die verschiedenen Dressurmethoden kennen zu lernen, die Genese 
der Dressurleistungen zu verfolgen und die Dressur-Dauer der verschiedenen Individuen und Arten miteinander zu vergleichen.

c) D i e $\mathrm{Zw}$ i s c h e $\mathrm{nfälle}$. Diese sind ja im Zirkus infolge des beschränkten menschlichen Einfühlungsvermögens in tierische Umwelten nicht allzu selten und für den tierpsychologischen Untersucher deshalb oft von besonderer Wichtigkeit, weil durch sie zuweilen gewisse latente Phänomene, die sonst überhaupt nie manifest werden, sich plötzlich in greller, blitzartiger Beleuchtung aufs deutlichste präsentieren, und zwar Phänomene, die oft ganz besonders aufschlussreich sind. Hier wären z. B. Situationen zu erwähnen, wo ein Dompteur aus irgendeinem Grunde angegriffen, von einem anderen Tier aber in wirksamer, unzweideutiger Weise verteidigt wird, wofür wir zahlreiche Belege haben. Ausserhalb dieses Zwischenfalles kann der Dompteur höchstens gefühlsmässig anhand verschiedener Anzeichen vermuten, dass das eine Tier gefährlich werden, das andere dagegen sich einmal als Beschützer erweisen wird. Erst der Zwischenfall selber zeigt dann, ob die gefühlsmässige Beurteilung gestimmt hat, was übrigens bei erfahrenen Dompteuren allermeist der Fall ist.

Es geht hier indessen nicht an, jede dieser drei Erscheinungsgruppen mit Beispielen zu belegen; das wird in der später erscheinenden Arbeit ausgiebig geschehen. Hier handelte es sich ja nur darum, grundsätzlich den wichtigsten Teil des Programmes aufzuzeigen, dem der Tierpsychologe bei der Untersuchung der Dressurphänomene im Zirkus zu folgen hat.

\section{LITERATUR.}

1935. Hediger, H. Zur Biologie und Psychologie der Zahmheit. Archiv ges. Psychol., Bd. 93.

1929. Menzel, R. und Menzel, R. Wesenserprobung, ihre theoretischen Grundlagen und ihre praktische Ausführung. Selbstverl. Ver. Dtsch. Schäferhunde (S.V.), Augsburg 3. (Zitiert von D. MüLLER, mir nicht vorgelegen.)

1930. _ Die Verwendung der Riechfähigkeit des Hundes im Dienste der Menschheit. Berlin.

1932. Müller, D. Wesensgrundlagen. Zs. f. Hundeforschung, Bd. 2.

1934. UехкёlL, J. und Kriszat, G. Streifzüge durch die Umwelten von Tieren und Menschen. Berlin. 


\section{$2 \mathrm{BHL}$ Biodiversity Heritage Library}

Hediger-Zurbuchen, H. 1935. "Zirkus-Dressuren und Tierpsychologie." Revue suisse de zoologie 42, 389-394. https://doi.org/10.5962/bhl.part.117667.

View This Item Online: https://www.biodiversitylibrary.org/item/148565

DOI: https://doi.org/10.5962/bhl.part.117667

Permalink: https://www.biodiversitylibrary.org/partpdf/117667

\section{Holding Institution}

American Museum of Natural History Library

\section{Sponsored by}

BHL-SIL-FEDLINK

\section{Copyright \& Reuse}

Copyright Status: In copyright. Digitized with the permission of the rights holder.

Rights Holder: Muséum d'histoire naturelle - Ville de Genève

This document was created from content at the Biodiversity Heritage Library, the world's largest open access digital library for biodiversity literature and archives. Visit BHL at https://www.biodiversitylibrary.org. 